\title{
Pesticide exposure may trigger food allergies
}

Published online: 05 October 2012

(C) Springer Healthcare 2012

medwireNews: Results from the US National Health and Nutrition Examination Survey (NHANES) suggest that high exposure to dichlorophenols, chemicals found in pesticides and used for chlorinating water, may increase a person's risk for developing food allergies.

Elina Jerschow (Albert Einstein School of Medicine, Bronx, New York, USA) and colleagues tested links between dichlorophenol exposure and allergic sensitization in 2211 people included in the US NHANES 2005-2006 aged 6 years and older.

Exposure to dichlorophenols was assessed by measuring urine levels and was defined as high if concentrations were greater than the $75^{\text {th }}$ percentile of the population or above.

Allergen-specific serum immunoglobulin E levels were use to measure the prevalence of food (eg, peanut, egg, milk, and shrimp) and environmental (eg, white oak, birch, mouse, and rat) allergies, with levels above $0.35 \mathrm{kU} / \mathrm{L}$ or higher deemed to indicate positive allergic sensitization. Following multivariable adjustment, people with high levels of dichlorophenols in their urine were a significant $80 \%$ more likely to be sensitized to at least one type of food.

However, no significant association between dichlorophenol exposure and sensitization to environmental allergens was observed.

Participants who had been exposed to two dichlorophenol metabolites or more were more likely to have one or more food allergies than those with no or less exposure, notes the team. They were also a significant $61 \%$ more likely to be allergic to a food and environmental allergen at the same time.

"In this population, we found consistent associations between high levels of dichlorophenol exposure and a higher prevalence of food allergies," write Jerschow and colleagues in the Annals of Allergy, Asthma and Immunology.

"Previous research indicated that both environmental pollution and the prevalence of food allergies are increasing in the United States. The results of this study suggest that these two phenomena might be linked," they add, although they concede that "further prospective studies would be necessary to confirm this link."

By Helen Albert, Senior medwireNews Reporter

Reference

Ann Allergy Asthma Immunol 2012; Advance online publication 